\title{
Holes Cannot Be Counted As Immaterial Objects
}

\begin{abstract}
In this paper I argue that the theory that holes are immaterial objects faces an objection that has traditionally been thought to be the principal difficulty with its main rival, which construes holes as material parts of material objects. Consequently, one of the principal advantages of identifying holes with immaterial objects is illusory: its apparent ease of accounting for truths about number of holes. I argue that in spite of this we should not think of holes as material parts of material objects. This is because the theory that holes are properties does not face the same difficulties as either of these theories that construe holes as objects of some sort.
\end{abstract}

\section{Introduction}

In their classic discussion of the ontology of holes, Holes and Other Superficialities [Casati and Varzi 1995], Casati and Varzi offer an account of holes as immaterial objects. In defence of this position they go to considerable lengths to show that it can provide satisfactory solutions to the various metaphysical puzzles raised by holes. They also provide a battery of arguments against the most developed materialist account of holes, given by David and Stephanie Lewis in their incisive and entertaining paper 'Holes' [Lewis and Lewis 1970], in which they offer an account of holes as a certain kind of material object: the material surfaces of material objects. More recently I have advanced the view that holes should not be construed as objects of any sort, neither material nor immaterial 
[Meadows 2011]. Instead, I suggest holes should be construed as properties or relations. In the present paper I intend to offer further arguments in support of this position. ${ }^{1}$

The debate over which account of holes should be preferred typically proceeds by considering, first, how much of our common-sense thinking about holes can be captured by the theory and, second, how ontologically economical it is. Casati and Varzi's account of holes as immaterial objects does well on the first criterion, but is more ontologically inflationary than David and Stephanie Lewis'. Their position, by contrast, is relatively ontologically economical, but runs into trouble in capturing our common-sense thinking about holes. As I have argued elsewhere [Ibid.], my own account of holes as properties is ontologically economical in the sense that it is no more inflationary than any position that acknowledges a categorial distinction between objects and properties at the level of fundamental ontology. My purpose here is to argue that there are some features of common-sense thinking about holes that both Casati and Varzi's position and David and Stephanie Lewis' position have difficulty capturing. Moreover, these difficulties are not shared by the account of holes as properties. Consequently, these considerations count as reasons in favour of that view.

A successful argument of this sort is important because it highlights a theoretical advantage of the property account over the view that holes are immaterial objects (hereafter the 'Immaterial Object Theory'). The strongest consideration in favour of the Immaterial Object Theory is that it provides a straightforward, unproblematic account of common-sense truths about the identity and number of holes in material objects. This is in contrast to its traditional competitor theories which construe holes as material surfaces of

\footnotetext{
${ }^{1}$ There are other accounts of holes that are on the market, including the idea that holes are conventional entities [Miller, 2007] or products of our inability to paraphrase talk about holes [McDaniel, 2010]. However, my concern here is principally to address realist conceptions of holes, which these accounts do not offer. My reason for this is simply a methodological one: that our common-sense conception of holes is realist and that such
} 
material objects, such as the Lewises' (hereafter the 'Material Part Theory'), which requires non-standard interpretations of crucial terms like 'inside', 'outside' and 'is the same hole as' to deal with such sentences. For instance, if I put my finger inside the hole in my wedding ring (as we might naturally say), the finger would be outside the material surface of the ring (as we might naturally say), but consequently outside the hole on the Lewises' account. In such cases the Lewises suggest assigning a non-standard meaning to terms like 'inside' and 'outside' [1970: 209].

Such a complication might be dismissed as simply the acceptable cost of a materialist account of holes, but in Holes and other superficialities Casati and Varzi press this objection more forcefully [1995: 29-30] by arguing that even granting these linguistic contortions the view still gets certain number claims concerning holes wrong. I will shortly discuss their argument for this in some detail, but it is important to emphasise that if Casati and Varzi's argument is cogent, then the Lewises' Material Part Theory appears to be accruing significant theoretical costs, which makes the Immaterial Object Theory look increasingly attractive in spite of its own theoretical costs. This is perhaps the most compelling case that is available for the Immaterial Object Theory. My intention in this paper is to argue that, on this score, this putative advantage of Casati and Varzi's position is illusory. As I will show, there are reasons for thinking that construing holes as immaterial objects is really no better at accounting for the identity and number of holes than its traditional Lewisian rival. Moreover, as I shall argue, the reasons for their inadequacy are surprisingly similar. However, as I have indicated, my ultimate purpose is not to defend the Lewises' view - my real purpose is to show that both the Immaterial Object Theory and the Material Part Theory have a significant disadvantage on this score that is not shared by the theory which construes holes as properties. In the final section of the paper I make the 
case for this theory in light of these difficulties with the Immaterial Object Theory and the Material Part Theory.

\section{Material Surfaces: Multiple Candidates For Identification}

Turning to Casati and Varzi's criticism of the Material Part Theory, the main obstacle, as they see it, to the view that holes are material surfaces is that any attempt to identify some particular superficial part of a material object with its hole is going to be arbitrary:

"... one must address the basic question of how one can identify holes with their linings, since it seems that a hole may have indefinitely many linings." [loc. cit.]

The idea here is that there are multiple candidates for identification with the hole, such as the single-atom thick superficial part, the two-atom thick superficial part, etc. We are owed an answer to the question, which of these is the hole, and on what principled grounds? As Casati and Varzi concede, the absence of a principled answer to this question is not an insurmountable problem, as the Lewises could (and did) resist the demand that we pick out one and only one of these as the hole. We could say, rather, that all of these candidates are holes, all non-identical, but nonetheless all the same hole. This, of course, requires us to assign a different meaning to the predicate 'being the same hole as' than it might naturally be thought of as having, which is just the identity relation. The Lewises' suggested alternative interpretation for the predicate is the relation 'having a common part that is itself a hole.' [1970: 209] 
However, this move brings with it further problems [Casati and Varzi 1995: 30]. Imagine two holes side by side in a single lump of matter, L, as shown in figure (1).

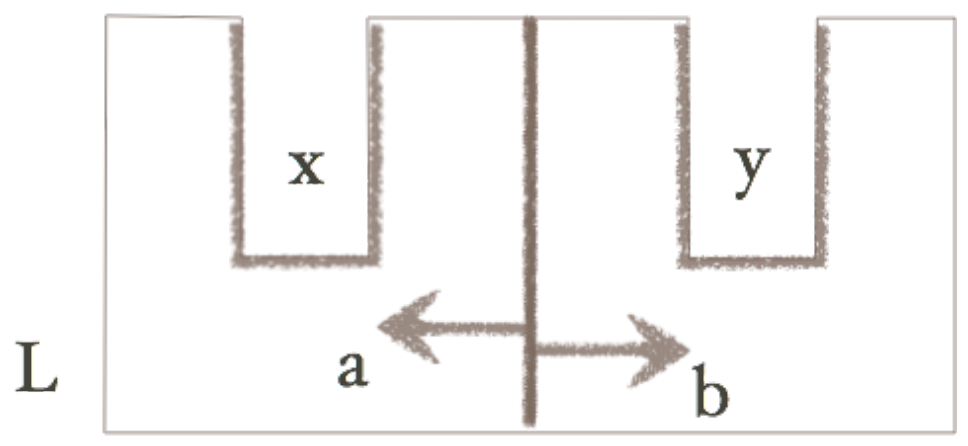

(Figure 1)

Because the relation 'being the same hole as' is just the relation of having a common part that is itself a hole, it turns out that the left half of the lump, $a$, is the same hole as the single atom thick part $x$ of that half: i.e. the skin of that half of the lump. Moreover, the right half of the lump, $b$, is itself the same hole as the part $y$ of $i t$ that is a single atom thick. However, the whole lump $L$ is also the same hole as both of these. This is problematic because the two single-atom thick holes are not the same hole, because they have no common parts. In which case, as Casati and Varzi point out, we have too many holes:

"But then the basic question remains: How many holes are there in the cube? Two or three? The Ludovician account seems to yield the second answer-and that is hardly tenable" [Loc. Cit.]

In light of Casati and Varzi's point that there are indefinitely many hole linings, they understate the problem in this quote. The Ludovician answer will not be simply 'three' - in fact, the answer will be 'indefinitely many'.

There is a response available at this point: to deny that the relation 'is the same hole as' is transitive. However, these adjustments away from common-sense ways of 
thinking and speaking about holes count against the reductionist account. Moreover, it still remains that the Lewises' view counts three holes where there should be two. In consequence, the initial attraction of construing holes as sui generis, immaterial objects is enhanced by these conceptual contortions. This far I agree with Casati and Varzi's objection to the Material Part Theory. However, I think that much the same problem arises for the Immaterial Object Theory, which impugns its credentials as the theory most able to capture our common-sense intuitions about holes.

\section{Multiple Candidates and the Immaterial Object Theory}

Recall the starting point of Casati and Varzi's objection to the Material Part Theory: the troubling observation was that there are indefinitely many candidates for identification with any particular hole. In the case of the Material Part Theory the problem arises because there are indefinitely many candidate material parts of the material object hosting the hole. But the very same sort of problem arises for the Immaterial Object Theory. According to this account, holes are immaterial objects constituted by, but not identical with, regions of empty space. But, of course, there are indefinitely many candidates which could be identified with any given hole. This is because there are indefinitely many empty regions of space that could be candidate constituents for any given hole. Consider a hole in the surface of a cube, as in figure 2(a). Now consider three ways in which such a hole could be filled: by filling the empty space so that the surface at the site of the hole becomes concave, as in figure 2(b); convex, as in figure 2(c); or flat and level, as in figure 2(d). So, there are three different regions of empty space that could count as the hole: and of course there are many more, such as a question-mark shaped region of space ballooning out of the hole. 


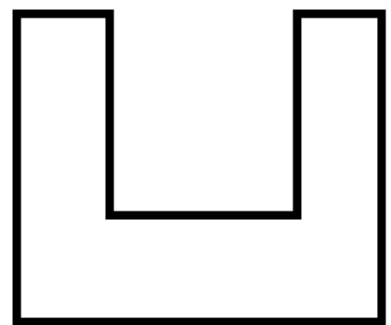

a

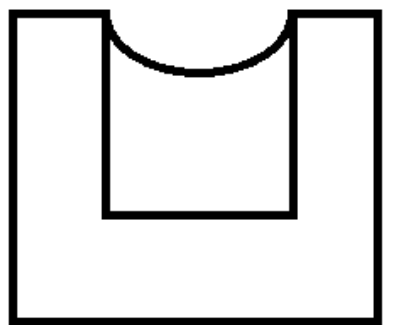

b

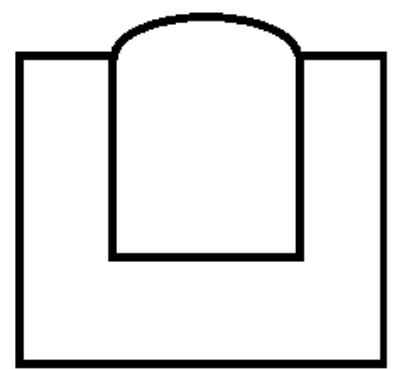

C

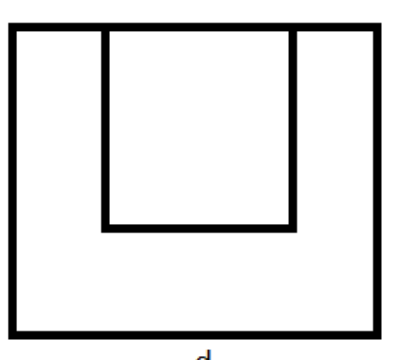

d

(Figure 2)

One objection at this stage would be to claim that among the regions of space demarcated in figure 2 there is really just one plausible candidate that can count as the constituent of the hole - figure 2(d). There is, after all, a natural, intuitive answer to the question of where the boundary of the hole is in figure 2: an answer that is reflected by the intuitive answers we might give under various circumstances to the question whether some material object is inside the hole. Were a material object lowered into the hole from above, it seems natural enough to say that the object will be inside the hole when it is within the boundary shown in 2(d), even when it is outside the boundary shown in 2(b). Equally, it seems intuitive enough to say that the object will be outside the hole when it is within the boundary shown in 2(c), if it is nonetheless outside the boundary shown in 2(d). So, it might be urged, our intuitions about the boundaries of the hole are justified by our intuitions about when an object is inside or outside of it. In a way, this is incontrovertible. ${ }^{2}$ However, there are reasons for being unsatisfied by a reply of this sort: firstly, it is a matter of what it is to be a boundary that it carries implications about the relations of inside and outside. So, it is no surprise that our intuitions about when things are inside or outside an entity with a boundary match our intuitions about their boundaries. However, because our intuitions about what is inside and what is outside are consequences of our intuitions about such

\footnotetext{
${ }^{2}$ I am grateful to an anonymous reviewer for suggesting this objection.
} 
boundaries, the former can hardly be thought to be independent, or principled justification of the latter.

Returning to Casati and Varzi's theory: as with the Material Part Theory, the problem for the Immaterial Object Theory becomes more acute when we consider the same kind of example discussed above in connection with the criticisms of the Material Part Theory: where there are two holes of the sort in a single lump $L$ side by side in a material object, as in figure 3 :

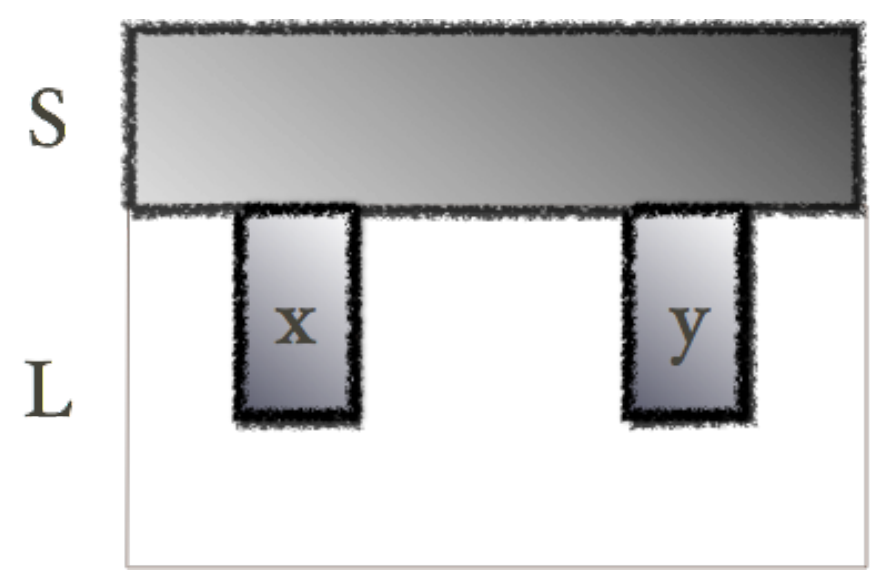

(Figure 3)

We have seen that there are multiple candidate constituents for each individual hole, including those with weird and whacky shapes. For any pair of these, $x$ and $y$, there will be at least one region of space $S$ that has $x$ and $y$ as parts. The question here naturally arises (as the analogous question arose for the Material Part Theory): on what principled grounds do we rule out this region of space $S$ as coextensive with a hole (or the regions of space in figure 2(b) or 2(c))? It should be noted that it will clearly not be adequate to simply observe in response that acknowledging $S$ as a hole is overly permissive relative to our common sense way of thinking about holes: it is precisely the permissiveness of the account that is problematic about it. The objection can be expressed in the following way: holes cannot be counted properly when construed as immaterial objects. 
One possible response here is to pursue the Lewisian strategy: the Immaterial Object Theory could hold that $S$ and $x$ are the same hole in the sense of having a common part that is a hole, but are non-identical, as are $S$ and $y$. But this has the same undesirable consequence as Lewis's Material Part Theory: viz. that by the transitivity of the 'is the same hole as' relation $x$ and $y$ will be the same hole, but because they have no common parts also cannot be the same hole. The same response is available for the Immaterial Object Theory of holes as was available for the Lewises: deny the transitivity of the 'is the same hole as' relation. But now it seems that the Immaterial Object Theory has the same disadvantage of the Material Part Theory here, but without the advantage of its ontological economy.

\section{Fillers and Holes}

I've argued that the Immaterial Object Theory shares a significant disadvantage with its traditional rival, the Material Part Theory, in being unable to capture common-sense number claims about holes. I want now to consider a line of response available to Casati and Varzi which has the potential to allow them to resist this objection. This response appeals to the notion of a 'perfect filler' for holes, a notion which they develop in the course of offering a classificatory system for holes. Their idea is that, just as the topologically relevant features of material objects allow us to separate them into classes, the feature of being fillable by certain material objects allows us to classify holes. Holes that can be filled by fillers of the same shape and size are to be classified as of the same kind. However, holes can be filled in multiple ways: a hole could be filled so as to be left with a depression; it could be filled so that it is level and continuous with the surrounding material; or it could be over-filled. Moreover, it could be filled in one of these ways, but so as to leave internal 
holes in the filler. In recognition of these points, Casati and Varzi introduce the idea of a 'perfect filler': a filler which fills holes exactly; no more, no less. This will clearly be of help in the face of my objection, because the perfect filler for a hole can be used as a proxy to identify exactly the extent of the unique region of space constituting any given hole, despite the fact that it could be under-filled or over-filled.

The idea of a perfect filler for a hole goes by way of the notion of a 'complete filler'. Their idea is that a complete filler is one which fills a hole so as to leave no holes, whether internal or not.

"A complete filler entirely fills the hole..., whereas a partial one does not...." [Casati and Varzi 1995: 57]

This is intended to capture the idea that some fillers are not sufficient to fully fill a hole, because they leave holes. However, many complete fillers of holes will, as we might say, 'over-fill' holes. Further, in the class of complete fillers for each hole most of the fillers will be parts of other complete fillers. However, for each kind of material that a filler could be composed of, there will be one complete filler which has no proper parts that are themselves complete fillers of the hole. These fillers, as we might say, completely fill the hole, but don't over-fill it. Such fillers Casati and Varzi call 'perfect fillers'.

"Now, imagine partitioning the class of all potential complete fillers of a hole (of a given shape and size) into the equivalence classes determined by the relation "is made of the same sort of stuff as." The greatest lower bound of each such class, relative to the partwhole relation, may be called a perfect filler of the hole: it fills up the hole completely, but without overflowing...." [Casati and Varzi 1995: 58] 
My objection to the Immaterial Object Theory was that it faces the same difficulty as the Material Part Theory in accounting for claims about number of holes. This is because there are multiple candidates for the immaterial objects to be identified with the holes. We are now in a position to see how Casati and Varzi could appeal to the notion of a perfect filler in reply: they could claim that perfect fillers for holes identify which immaterial object is the hole. ${ }^{3} \mathrm{~A}$ hole will be just that immaterial object which is constituted by the region of space filled by its perfect filler.

The idea is that we take any material object $m$ with a hole $h$. By Casati and Varzi's lights, $h$ is identified by its perfect filler: the filler that leaves no holes and has no part that is itself a complete filler. This is meant to rule out fillers that would naturally be described as 'over-filling' the hole. However, it's not clear that this account can rule these fillers out. Take, for example, the material object shown in figure 2(a). Casati and Varzi's account aims to eliminate fillers like those shown in $2(b, c)$. There is only one candidate for the hole in $m$, because only the filler shown in $2(d)$ counts as a perfect filler. The hole in $m$ extends up to, but no further than, the boundary of the filler shown in 2(d).

This solution will only be successful if it can be demonstrated that the filler shown in $1(c)$ is not a perfect filler. It is not a perfect filler if it has a part that is itself a complete filler. But on what grounds do we say that the filler shown in 1(c) has a part that is a complete filler?

Intuitively the answer to this is that 2(c) has filler 2(d) as a part, which is itself a complete filler for the hole. But then the question arises: why is 2(d) a complete filler for the

\footnotetext{
${ }^{3}$ It should be made clear, though, that Casati and Varzi themselves do not employ their account of perfect fillers to deal with the counting problem l've identified here. It is at least logically possible that a reply to the counting problem could be independent of an account of our intuitions about which of the regions of space marked out in figure 1 is coextensive with the hole.
} 
hole? Here again there seems to be an intuitively compelling answer: $2(d)$ is a complete filler because it fills the hole, but leaves no holes. The problem is that this is true only if the region of space filled by part $p$ of $2(c)$, where $p=(2(c)-2(d))$, is not part of the hole. So, the question boils down to this: is the region of space filled by $p$ a part of the hole?

The most natural inclination is to answer this question in the negative: the region of space filled by $p$ is not part of the hole. But on what grounds do we say this? The justification for not counting $p$ as part of the hole does not seem to be the account of holes supplied by Casati and Varzi: to put Casati and Varzi's notion of perfect fillers to use, we already need to have an answer to this question. Moreover, we clearly cannot simply appeal to our intuitions at this point. This is because it is a measure of the adequacy of an appeal to perfect fillers that it agrees with our intuitions. Appealing to our intuitions here would render vacuous any agreement between the theory offered by Casati and Varzi and our intuitions. So, in spite of all Casati and Varzi have said about the relationship between perfect fillers and holes, the space filled by the filler shown in 2(c) is a candidate for the hole. This point generalises: given Casati and Varzi's account, for any hole $h$ in any material object $m$ the perfect filler identifies the hole, but there are multiple candidates for the perfect filler. Consequently, there are multiple candidates for identification with the hole.

There are other reasons for thinking the account not helpful in limiting the candidates for holes. Take a mountain with a naturally formed cave, $c$, as in figure 4: 


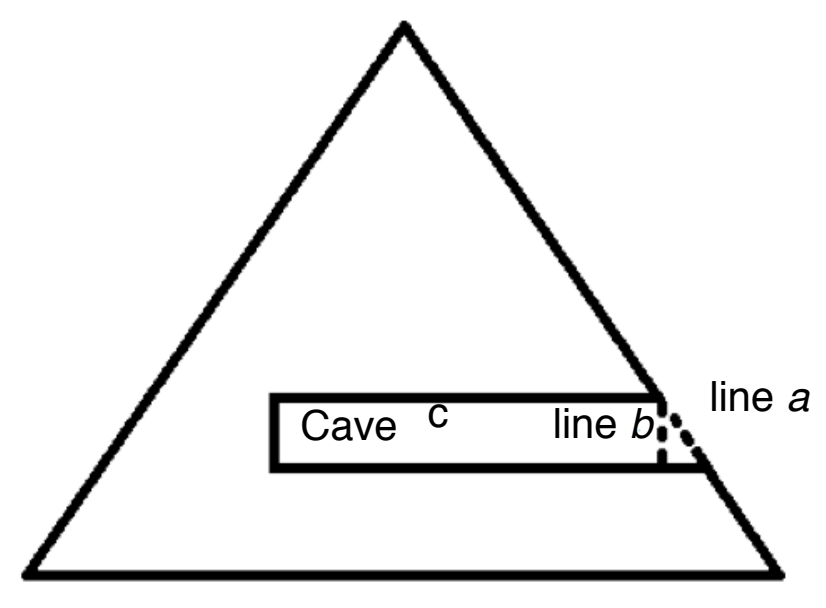

(Figure 4)

This case is interesting because, in addition to all the complete fillers that are not perfect fillers, there are two plausible candidates for the complete filler for $c$ : i.e. $c$ 's perfect filler. The first follows the line of the mountainside and fills up to line $a$; the second fills the the vertical line $b$, creating a cliff. Pace the foregoing argument, there is little controversy that the filler to line $a$ is a complete filler. However, the filler to line $b$ requires a little comment. If $c$ is formed by excavating from line $a$, then the filler to $b$ is less plausibly construed as a complete filler for the hole. This is because, in such a situation, the section from line $a$ to line $b$, is part of the excavated hole $c$. However, if $c$ is formed just by the sedimentation of the earth when the mountain is formed, then the filler to $b$ is as plausible a candidate as the filler to $a$ for the complete filler for $c$. In response, it might be urged that the filler to $b$ leaves a hole: a hole extending from line $b$ to line $a$. So, by Casati and Varzi's lights, the filler to line $b$ cannot be a compete filler. Consequently just one of these fillers is a plausible candidate for a complete filler.

The first step in replying to this line of argument is to accept that the filler to $b$ leaves a hole, but to observe that it is an open question whether this hole is a part of $c$. I said that in those situations where $c$ is excavated from a mountain with no holes, then the hole from line $b$ to line $a$ is clearly a part of $c$. However, in situations where $c$ is formed just by the 
sedimentation of the earth as the mountain is formed, this is less intuitively obvious. So, there is a distinction to be drawn between those fillers that leave holes that are parts of the hole they are fillers for, and those fillers that leave holes that are not parts of the holes they are complete fillers for. These latter fillers have as much right to be construed as complete fillers for their holes as do fillers that leave no holes whatever. The idea is that Casati and Varzi's category of complete filler does not cut finely enough: it is too blunt a tool. Recognizing this distinction allows the following reply to the foregoing objection: it is clear that the filler to $b$ leaves a hole, but it leaves a hole that is not part of $c$. This is compatible with it being a complete filler for $c$, because it is one of those complete fillers that leave a hole which is not part of the hole it fills.

The upshot is that it remains that there are two equally plausible, incompatible candidates for the complete filler for $c$. However, it's important to note that this case is special: the plausibility of the filler to line $b$ as a candidate for the complete filler for $c$ relies on the orientation of the material object to the gravitational centre of the planet. This is somewhat disconcerting, as it is distinctly odd to think that whether something is a part of a hole depends on the orientation of the hole. Resistance to the idea that facts about holes depend on their orientation might lead someone to argue in this way: there is no metaphysically significant difference between $c$, with its candidate complete fillers to lines $a$ and $b$, and the hole in figure 2, with its candidate complete fillers shown in 2(b) and 2(d). We think that $2(\mathrm{~b})$ is not really a candidate complete filler for the hole in figure 2; so, by parity of reasoning, the filler to line $b$ is not really a candidate for a complete filler for $c$.

The problem with this line of argument, of course, is that it is possible to argue, by parity of reasoning, in the opposite direction: from there being two candidates for complete fillers for $c$, together with the metaphysical insignificance of the orientation of holes, to the conclusion that $2(b)$ is a candidate complete filler for the hole in figure 2 . So, it appears 
from the foregoing discussion that an appeal to the notion of a complete filler will not be particularly helpful to the Immaterial Object Theory in warding off the worry that there are multiple candidates for any hole, and the consequent objection that the theory cannot account for common-sense truths about number of holes.

\section{Conclusion}

So, it seems that the Immaterial Object Theory fares no better than its rival, the Material Part Theory, when it comes to accounting for truths about numbers of holes. However, on reflection, this should not be a surprise. Both positions individuate holes by appeal to entities that can stand in mereological relations to other objects of the same sort. For the Material Part Theory, holes are identified with material objects that can be parts of other material objects; for the Immaterial Object Theory, holes are constituted by regions of space that are parts of other regions of space. The existence of mereological relations between these entities is precisely why, in each case, these are plausible candidates for identification with the hole. After all, if regions of space or material parts are not merelologically related, this lessens the plausibility of the claim that both are candidates for identification with the hole. For example, the region of space bound by my wedding ring is a candidate owing to its location relative to my ring, but the region of space occupied by my computer is not. A connected region of space partly composed by both, however, is a candidate because it contains the region bound by the ring as a part. The challenge for both theories is to offer a principled reason for preferring to identify this part with the hole, rather than some mereological extension of that part.

Earlier I remarked that because both theories share the disadvantage of being unable to account for common-sense truths about number of holes the Lewises' position 
looks the more attractive one, because it at least has the advantage of a sparser ontological economy than Casati and Varzi's position. If these were the two principal candidate realist theories, then the Material Part Theory should be preferred to the Immaterial Object Theory. However, I think it would be too quick to leave the matter there. In my recent paper [Meadows, 2011] I argued that there are reasons to think that it is a mistake to construe holes as objects of any sort at all, which is a general type of position I call 'objectualism'. Instead, we should construe holes as properties, rather than objects, which I call 'non-objectualism'. My argumentative strategy there was to argue directly for non-objectualism from the ontological status of angles, which raise all the same metaphysical puzzles as holes. But my discussion there did not offer a comparison of the relative merits of that position compared with the traditional alternatives of the Immaterial Object Theory and the Material Part Theory. We are now in a position to make such a comparison.

One of the most significant disadvantages of the Material Part Theory is that it is unable to account for common-sense truths about holes. Where two holes are side by side in a lump of matter, the position cannot make the relevant claim about number without significant revisions to the meanings of the terms involved. The most compelling case for preferring the Immaterial Object Theory was that it can effortlessly deal with such claims without any revision to the meanings of crucial terms. In this way the ontological 'extravagance' of immaterial objects is justified. But the foregoing argument shows this explanatory advantage of the Immaterial Object Theory is illusory: in fact both theories encounter the same difficulty accounting for claims about number of holes. However, this difficulty will not be shared by an account of holes that construes holes as properties. The reason for this is that properties do not stand in mereological relations to other properties. As explained above, the reason that there are multiple candidates for holes by the lights of 
either the Immaterial Object Theory or the Material Part Theory is that there are candidate objects of these sorts that stand in mereological relations to other candidate objects of these sorts. This is simply not the case for the account of holes as properties developed in my earlier paper, because properties don't stand in mereological relations to other properties.

At the outset it seemed that the most compelling argument for the Immaterial Object Theory was that it can effortlessly account for truths about number of holes. It turns out that this theoretical advantage is really possessed by the theory that construes holes as properties. Moreover, as I have argued elsewhere, [Meadows, 2011] this kind of theory can explain a wide range of truths about holes, such as the existential dependency of holes on the material objects they are holes in, and the existential independence of holes from the matter filling them. However, in the interests of full disclosure, it is important to point out that there is one kind of common-sense truth about holes that this account encounters difficulties with: that holes can survive changes in size and shape. One way of seeing this problem is to construe holes as relations between material parts of material objects. If a hole is something like a relation between material parts of the object they are in, then when the shape or size of the hole changes, the relations between those parts cease to be instantiated and other relations become instantiated. Then, if holes just are those relations, holes cannot survive changes of size and shape. Holes persist only as long as they undergo no such changes: such changes involve the destruction of the holes and the creation of a new one.

I have two reservations with this line of argument. First, it is not clear that we must construe properties as relations, rather than monadic properties. Secondly, it is not clear that even if we do construe properties as relations, then we must construe them as the spatial relations between material parts of the material object. We could instead construe 
them as numerically distinct relations that are existentially dependent on those spatial relations. However this may be, further work needs to be done to spell out in detail the right approach here. However, for present purposes the important point is the following one: this theoretical disadvantage of the theory is not shared by the Immaterial Object Theory; but, this is more than compensated for by the theoretical advantage of being able to account for truths about number of holes. So, on balance, the view that construes holes as properties is to be preferred to both of its rivals: the Material Part Theory and the Immaterial Object Theory.

\section{Bibliography}

Casati, R. and Varzi, A. 1995. Holes and Other Superficialities, Cambridge: MIT Press.

Lewis, D. and Lewis, S. 1970. Holes, Australasian Journal of Philosophy. 48/2: 206-212.

McDaniel, K. 2010. Being and Almost Nothingness, Noûs 44 (4):628-649.

Meadows, P. J. 2013. What Angles Can Tell Us About What Holes Are Not, Erkenntnis 78 (2): 319-331.

Miller, K. 2007. Immaterial Beings, The Monist 90 (3):349-371

Wake, A., Spencer, J. and Fowler, J. 2007. Holes As Regions of Spacetime, The Monist 90 (3):372-378 